\title{
La religión institucionalizada en las federaciones deportivas. Análisis antropológico de los vínculos entre el taekwondo y las religiones orientales
}

\author{
Javier Eloy Martínez-Guirao* (D) \\ Universidad de Murcia (Spain) \\ Recepción: 11/06/2018; Aceptación: 12/01/2019; Publicación: 14/01/2019.
}

\section{Resumen}

El taekwondo se ha popularizado desde hace décadas y su práctica ha pasado a formar parte de los países occidentales. Una de sus líneas de expansión e introducción en Occidente, al igual que otras artes marciales, fue la esfera filosóficoreligiosa, que ha sido impulsada desde las propias federaciones deportivas, $\mathrm{y}$ ha enfatizado aspectos relacionados con el taoísmo, el budismo, el confucianismo y las religiones nacionales coreanas. Nos basamos en un estudio de fuentes documentales, contextualizado dentro de una investigación etnográfica, para analizar los elementos religiosos que aparecen en la cultura material, valores, técnicas y prácticas que se han desarrollado o se desarrollan en los gimnasios, así como las exégesis simbólicas que se realizan desde las instituciones oficiales.

Palabras clave: Antropología; taekwondo; religión; filosofía; símbolos: artes marciales; deportes de combate.

\begin{abstract}
Institutionalized religion in sports federations. Anthropological analysis of the links between taekwondo and Eastern religions

\section{Abstract}

Taekwondo has been popular for decades and its practice has become part of Western countries. One of its lines of expansion and introduction in the West, like other martial arts, was the philosophical-religious sphere, which has been promoted by the sports federations, and has emphasized aspects related to Taoism, Buddhism, Confucianism and Korean national religions. I rely on a study of documentary sources, contextualized within an ethnographic investigation, to analyze the religious elements that appear in the material culture, values, techniques and practices that have been developed in the gymnasiums, as well as the symbolic exegeses that are made from official institutions.
\end{abstract}

Keywords: Anthropology; taekwondo; religion; philosophy; symbols; martial arts; combat sports.
A religião institucionalizada nas federações esportivas. Análise antropológica dos vínculos entre o taekwondo e as religiões orientais

\section{Resumo}

O taekwondo é popular há décadas e sua prática tornou-se parte dos países ocidentais. Uma de suas linhas de expansão e introdução no Ocidente, como outras artes marciais, foi a esfera filosófico-religiosa, promovida pelas federações esportivas, e enfatizou aspectos relacionados com o taoísmo, o budismo, o confucionismo e as religiões nacionais coreanas. Nesta análise, baseamo-nos no estudo de fontes documentais, contextualizadas no âmbito de uma investigação etnográfica, para se analisarem os elementos religiosos que surgem na cultura material, nos valores, nas técnicas e nas práticas que se desenvolveram e se vão desenvolvendo nos ginásios, assim como as exegeses simbólicas que se realizam nas instituições oficiais.

Palavras-chave: Antropologia; taekwondo; religião; filosofia; símbolos; artes marciais; desportos de combate.

\section{Introducción}

Las artes marciales procedentes de Asia Oriental se han popularizado desde hace décadas y su práctica ha pasado a formar parte de los países occidentales ${ }^{1}$. En muchas de sus ciudades se ofertan este tipo de actividades, junto a otras deportivas y gimnásticas, dentro del sistema de

\footnotetext{
${ }^{*}$ E-mail: j.eloymartinez@um.es

${ }^{1}$ La introducción de las artes marciales de Asia Oriental en diferentes países occidentales comienza en la segunda mitad y, especialmente, a finales del siglo XIX (Gutiérrez-García, 2006, 2007; Gutiérrez-García, PérezGutiérrez, Acevedo, \& Cheung, 2010). En España, a partir de la década de 1950, comienza a practicarse el judo (Villamón \& Brousse, 1999, p. 130; Gutiérrez-García, 2004). El kárate y el taekwondo llegarían a mediados de la década de 1960, pero será la década de 1980 cuando se popularicen y proliferen los gimnasios dedicados a estas y a otras artes marciales asiáticas (Martínez Guirao, 2011, pp. 126-131).
} 
valores que sustenta el culto al cuerpo actual. El taekwondo, que llegó a España, desde Corea, a mediados de la década de 1960, es una de las que tiene un mayor número de practicantes².

Si bien, hoy en día, en culturas donde el deporte es hegemónico, las artes marciales se han visto obligadas a adaptarse y configurarse como deportes (Martínez Guirao, 2011; Moenig, 2015) ${ }^{3}$, no podemos obviar que una de sus líneas de expansión e introducción en Occidente fue la esfera filosófico-religiosa, con la "construcción" y posterior "destrucción", en algunas de ellas, de un imaginario que enfatizaba aspectos relacionados con las religiones del taoísmo, el budismo y el confucianismo ${ }^{4}$.

La construcción de este imaginario tuvo lugar, en el caso del taekwondo, a través de tres vías interconectadas, vinculadas al mismo tiempo con la propia comercialización de estas artes marciales en las sociedades occidentales, donde trataron de cubrir de algún modo los espacios que dejaban la crisis de las religiones tradicionales y el materialismo predominante (Donohue, 1994; Cynarski, 2001, 2004; Cynarski, Sieber, \& Litwiniuk, 2006, p. 4; Martínez Guirao, 2011; Ferreira, Sonoda-Nunes, De Almeida, De Oliveira, \& Marchi Junior, 2012; Moenig, 2015). La primera se refiere a la proyección de su imagen desde el cine y la televisión, que presentaba a los artistas marciales como seres dotados de unas cualidades sobrehumanas y un poder extraordinario, unidos a la práctica religiosa y a los mensajes filosóficos (Martínez Guirao, 2010a, 2011; Bowman, 2010, 2013) ${ }^{5}$. La segunda vía tuvo que ver con la propia configuración de la práctica: sus lugares y espacios habilitados con su cultura material y símbolos, los ejercicios que se realizaban en ellos y la figura del maestro. La tercera, le confirió la oficialidad en la creación de discursos, que partían de las federaciones deportivas, desde Corea, y se exportaron al resto del mundo.

La construcción oficial de todo este imaginario se produjo desde la década de 1950, en la propia creación del taekwondo, tras la retirada de las tropas japonesas del territorio coreano. Las influencias de artes marciales japonesas como el kárate son evidentes, además de otras artes marciales chinas en las que estaban formados los maestros (Capener, 1995; Burdick, 1997; Dohrenwend, 2000; Martínez Guirao, 2011; Moenig, 2015). No obstante, se le trató de atribuir una continuidad histórica con actividades consideradas "tradicionales" en Corea como el taekyon y el subak, con los guerreros Hwarang del reino de Silla (57 a.C.-668 d.C.), y conexión con religiones igualmente nacionales. Desde esta perspectiva se podría considerar al taekwondo una "tradición inventada" (Moenig \& Kim Min-ho, 2016; Capener, 2016), en el sentido de Hobsbawn (2002).

El taekwondo llegó a España como un conjunto de técnicas, métodos de instrucción, valores, símbolos y mensajes filosóficos sincréticos procedentes de los sistemas religiosos del taoísmo, budismo, confucianismo y las religiones nacionales coreanas; parte de esa tradición que se pretendió construir (o reconstruir según los discursos oficiales). En este trabajo se va a hacer un

\footnotetext{
2 Según los datos del Consejo Superior de Deportes (2017) sobre licencias federativas, en el año 2017 el taekwondo era el tercer arte marcial en España en número de practicantes federados (con 43.007) y clubes inscritos (763), tras el judo (105.388, distribuidos en 1.025 clubes) y el kárate (70.290, en 1.431 clubes).

3 Aspecto que es compartido internacionalmente en un mundo globalizado, e institucionalizado en las federaciones deportivas y en la inclusión de algunas de estas artes marciales, como es el caso del taekwondo, en los propios Juegos Olímpicos.

4 En este sentido, para el caso español, véase el estudio de Pérez-Gutiérrez, Brown, Álvarez-Del-Palacio y Gutiérrez-Garciá (2015).

${ }^{5}$ Ejemplos de ello son la serie de televisión Kung Fu, el cine de Hong Kong o la saga de películas de Karate Kid. Aunque el taekwondo nunca fue presentado en este sentido explícitamente por los medios de comunicación y la industria cinematográfica, exceptuando en España dos breves menciones en el No-Do español de los años 1970 y 1976 (RTVE, 2018), y otra, más tarde, en el cine norteamericano con Campeón de campeones (1987) (con título original Best of the Best), durante aquellos años era conocido más bien como una versión del kárate, y en los imaginarios culturales se tendían a asemejar entre ellas a las artes marciales procedentes de Asia Oriental. Un análisis en mayor profundidad sobre las repercusiones de la industria cinematográfica en la introducción del taekwondo en España y sus vínculos con las religiones puede consultarse en Martínez Guirao (2010a, 2011). También para el caso español es interesante el artículo de Jiménez-Landaruzi, GomezAlonso, Izquierdo, \& Gutierrez-Garcia (2016), sobre las artes marciales y los deportes de combate en el NoDo. Sobre la influencia del cine de artes marciales en los imaginarios culturales de Occidente destaca análisis pormenorizado de las obras de Bowman $(2010,2013)$.
} 
análisis de la presencia filosófico-religiosa en el taekwondo, centrándonos en la simbología que aparece o aparecía en los espacios de práctica, en la indumentaria, en los ejercicios y en los discursos de los manuales y de las federaciones deportivas que tratan de hacer una exégesis de todos estos aspectos.

\title{
2. Las artes marciales como tradiciones inventadas
}

\author{
Para Hobsbawn (2002, p. 8),
}

La "tradición inventada" implica un grupo de prácticas, normalmente gobernadas por reglas aceptadas abierta o tácitamente y de naturaleza simbólica o ritual, que buscan inculcar determinados valores o normas de comportamiento por medio de su repetición, lo cual implica automáticamente continuidad con el pasado. De hecho, cuando es posible, normalmente intentan conectarse con un pasado histórico que les sea adecuado (...). Sin embargo, en la medida en que existe referencia a un pasado histórico, la peculiaridad de las "tradiciones inventadas" es que su continuidad con este es en gran parte ficticia.

El estudio de las artes marciales como tradiciones inventadas es algo recurrente en la actualidad, y no exclusivo del taekwondo. Por ejemplo, Bowman y Judkins (2016) coordinaron un número de la revista Martial Arts Studies con el título "The Invention of Martial Arts", en el que diversos autores analizaban desde esta perspectiva, entre otras, artes marciales y sistemas de lucha como el xilam (Jennings, 2016), el tabuik (Mason, 2016) o el aikido (Lefebvre, 2016).

Según Bowman y Judkins (2016, p. 1), la invención de las artes marciales puede darse en diferentes ámbitos y perspectivas, desde los mitos de origen, el folclore y las imaginaciones culturales populares de la tradición, hasta intervenciones o invenciones motivadas política e ideológicamente, u orientadas a la mercantilización, mediatización y espectáculo. Y se puede analizar en aspectos como los mitos y narraciones del linaje de las artes marciales consideradas tradicionales; en su redescubrimiento y reconstrucción; en la apropiación de las artes marciales desde discursos nacionalistas y, por tanto, en la construcción de la nación, gestión social y otros procesos políticos; en la difusión, desarrollo y transformación de las artes marciales; en su mercantilización y comercio internacional; en sus manifestaciones en diferentes contextos, etc. Como señala Bowman (2015, pp. 3-4), a diferencia de la permanencia y atemporalidad del mito, en realidad la historia está formada por discontinuidades, rupturas, revisiones, revoluciones, reconstrucciones, reinstalaciones y reimaginaciones.

Aproximarnos a las diferentes artes marciales como tradiciones inventadas, inmersas además en un proceso de globalización, nos permite comprender cómo se han adaptado a distintos contextos y el papel que la religión ha ido adquiriendo en ellas. Brown, Jennings y Molle (2009) han analizado las diferentes configuraciones que ha adquirido la religión en el desarrollo de las artes marciales en la actualidad. En algunas, como el tai chi, el repertorio técnico está directamente influenciado por el taoísmo, y ciertos estudios demuestran la importancia del $k i$ en su práctica (Frank, 2000, 2006). El zen puede relacionarse con la idea de la meditación en movimiento en la práctica de determinados ejercicios o disciplinas como el tiro con arco, y el aikido es vivenciado por algunos practicantes como un ejercicio espiritual. Por otro lado, abordan la idea de las artes marciales como religiones seculares, lo cual consideramos que llega a trascender la propia construcción o reconstrucción que busca vínculos con el pasado y con la tradición cultural, y es más bien una forma de adaptarse a las características y asimilarse en sociedades occidentales, enlazando con la diferentes subjetividades o necesidades de espiritualidad que se pueden dar.

El taekwondo surge tras la Segunda Guerra Mundial, tras décadas de dominio colonial japonés sobre tierras coreanas, con una política etnocida que prohibió la práctica de las actividades autóctonas, incluidas las artes marciales. Hasta entonces existían precedentes como el taekyon, el subak o el kwonbop que se practicaron en diferentes épocas históricas. Desde entonces, comenzaron a surgir escuelas que enseñaban artes marciales con denominaciones diversas ${ }^{6}$, y con

\footnotetext{
${ }^{6}$ Las escuelas Chongdokwan y Mudukkwan enseñaban un arte marcial que denominaban tangsudo, la escuela Chidokwan el kongsudo, la Mudukwan y la Changmukwan, el ju an pa kwonbop, y la Songmukwan el kongsudo (Martínez Guirao, 2011, p. 111).
} 
claras influencias del kárate y el chuan fa. Tras el paréntesis de la guerra de Corea (1950-1953), nuevas escuelas comenzaron a proliferar, junto a la idea de la necesidad de homogeneizar las diferentes artes marciales que impartían en ellas. Comenzaría entonces un proceso de "construcción" o "reconstrucción" del taekwondo, a partir de las disciplinas que se practicaban y de la recuperación de las artes marciales anteriormente existentes en Corea. Este proceso daría lugar a la creación de la KTA (Korea Taekwondo Association), que no incorporaría oficialmente el nombre de "taekwondo", según Capener (1995), hasta 1965, e intentó enfatizar su carácter nacional e histórico obviando las influencias externas y las discontinuidades históricas (Martínez Guirao, 2011, pp. 102-126).

\section{Vínculos históricos del taekwondo con las religiones}

Desde un punto de vista histórico se buscaron conexiones con restos arqueológicos, como pinturas realizadas en tumbas reales o estatuas que aparecen delante de templos, con cuerpos de guerreros de otras épocas históricas, con manuales de sistemas de lucha e incluso con los mitos de origen de la nación, y los vínculos con las religiones adquirieron importancia en la construcción simbólica y ritual y en la inclusión de valores. Algunas de estas conexiones se han mostrado incoherentes y como tales han sido criticadas.

Así, por ejemplo, uno de los manuales ilustrado más antiguos al que hacen referencia los discursos oficiales, el Muye dobo Tongji, que se remonta a al siglo XVIII, a pesar del énfasis que se ha tratado de poner en su continuidad con el taekwondo, lo cierto es que demuestra una enorme distancia entre entre el sistema de lucha que contiene y el taekwondo actual. Otro ejemplo es el del reino de Silla (57 a.C.-668 d.C.) y los guerreros Hwarang, periodo que se han difundido como de gran importancia en la historia antigua del taekwondo, donde además estuvo vinculado directamente a la religión. A este cuerpo de guerreros se lo ha relacionado con el chamanismo, el confucianismo, el taoísmo y el budismo. El código ético y moral en el que se basaban se suele atribuir al monje budista Wonkwan Popsa, y algunos autores (McBride, 2010) afirman que fue frecuente la instrucción por parte de monjes budistas a este grupo. Otros autores trazan en ellos incluso el posible origen del código bushido de los samuráis (Draeger \& Smith, 1969, p. 72). Sin embargo, autores como Moenig y Kim (2016) cuestionan los conocimientos que nos han llegado sobre los guerreros Hwarang, afirmando que gran parte de ellos se basan en meras especulaciones, lo que los lleva al terreno de la leyenda y el mito. Como afirman estos autores "La promoción de los hwarang como un grupo de guerreros ha tenido un éxito notable, a pesar del hecho de que no existen artefactos tangibles relacionados con ellos, como armas, armaduras, pinturas o documentos, en comparación, por ejemplo, con los registros históricos de los samuráis japoneses" (p. 157).

No obstante, lo cierto es que la relación real entre la religión y las artes marciales coreanas ha sido al menos tan discontinua como la de la propia importancia que cada una de las religiones ha podido tener en los distintos contextos históricos y culturales. Durante algunas épocas ciertas religiones se convirtieron en oficiales y otras fueron prohibidas, del mismo modo que las artes marciales adquirían auge o caían en decadencia según el periodo histórico (Martínez Guirao, 2011, pp. 73-108).

Pero la historia del taekwondo que contemplamos en este texto es otra mucho más reciente. Se remonta setenta años atrás, cuando el taekwondo se creó o se reconstruyó, y sobre todo desde su llegada a España, a partir de 1965, y su popularización en décadas posteriores (especialmente desde 1980). En sus inicios en España tendría importancia la diferenciación impulsada desde las fuentes oficiales entre taekwondo "tradicional" y taekwondo "moderno", que dio lugar a la dicotomía emic entre gimnasios "tradicionales" y gimnasios "modernos". Los primeros, entre otros aspectos, daban más importancia a la práctica de los pumses (poomsae) y mantenían unos vínculos más claramente establecidos con las religiones. En efecto, se trataba de gimnasios que daban una mayor importancia a los rituales, los símbolos, la veneración al maestro, etc. Los segundos prestaban más atención al combate y a la competición, con una mayor predominancia de los aspectos deportivos (Martínez Guirao, 2011, pp. 188-196). En la actualidad esta división conceptual está cada vez más desdibujada, con el auge del culto al cuerpo, la proliferación de los centros deportivos que desarrollan todo tipo de actividades, y la "creación" del "arte deportivizado", que se manifiesta en las competiciones de pumses. 


\section{Metodología}

Nos basamos, por un lado, en el estudio y análisis de fuentes documentales, como las páginas web de la Korea Taekwondo Association (KTA), la Kukkiwon? ${ }^{7}$, la World Taekwondo (WT) ${ }^{8}$ y los manuales, avalados por estos organismos, Taekwondo. Fundamentos y Pumses, de Kim Boo Hyang (1989), y Taekwondo dinámico, de Kyong Myong Lee (2000); el primero, presente en los gimnasios durante varias décadas, el segundo, con gran proyección internacional. Tenemos también presente el libro oficial de la Kukkiwon (2012), Taekwondo Textbook, que suele ser referencia en los estudios académicos sobre el taekwondo, y que coincide con los contenidos promulgados por la página web de la Kukkiwon, aunque el hecho de que sólo esté publicado en lengua inglesa y coreana dificulta su entrada en los gimnasios del contexto analizado. Todos ellos incluyen un discurso, construido e impulsado desde las instituciones oficiales, donde la religión está presente, como se expondrá a continuación.

Partiendo de una línea cualitativa, la información obtenida de estas fuentes documentales se analiza e interpreta de manera holística a partir de su significado (Corbetta, 2007, p. 389), de la misma manera que aquella que pudiera haberse obtenido de una entrevista u observación (Erlandson, Harris, Skipper, \& Allen, 1993, p. 99), pues como señalan algunos autores, "a todos estos 'textos', en realidad se les puede 'entrevistar' mediante preguntas implícitas y se les puede 'observar' En este caso la lectura es una mezcla de entrevista/observación y puede desarrollarse como cualquiera de ellas" (Ruiz Olabuénaga \& Ispizua, 1989, p. 69).

Entendemos que la información obtenida debe ser analizada teniendo en cuenta su contexto social y cultural de producción, y a partir de aquí de su intencionalidad, por lo que para comprenderla se han de tener en cuenta elementos culturales, históricos y nacionales. Por ello tenemos presentes las características de los sistemas de creencias propios de las culturas de origen, la política etnocida sobre Corea que se produjo por parte de Japón durante la primera mitad del siglo XX, el uso del taekwondo como parte de la reconstrucción o construcción histórica, nacional e identitaria, así como los diferentes procesos en los que se ha visto inmerso a lo largo de los años como la "coreanización", la "deportivización", la "globalización" y "glocalización", y diferentes "procesos de asimilación y adaptación cultural". Igualmente ha de ser analizada relacionándola con posicionamientos teóricos como las tradiciones inventadas (Hosbawn, 2002).

Partiendo de estas ideas, el análisis de los textos que contienen los discursos oficiales se ha realizado clasificando la información, para su posterior interpretación, en base a una serie de categorías analíticas relacionadas con la religión, es decir: 1) Otras formas de referirse al término religión: filosofía, espiritualidad, forma de vida, etc.; 2) los diferentes tipos de religiones o sistemas de creencias: budismo, taoísmo, confucianismo, religiones coreanas, y; 3) Elementos que aparecen en los relatos, la cultura material y las prácticas: símbolos, mitos, rituales y códigos de valores incluidos o relacionados con las religiones.

Por otro lado, contextualizamos este artículo dentro de un trabajo de campo etnográfico con observación participante realizado en los gimnasios de la provincia de Alicante ${ }^{9}$, en España. Esta investigación, más amplia, analiza categorías como el arte, el deporte, la defensa personal y la religión dentro de un proceso de asimilación y cambio cultural. En diferentes publicaciones derivadas de ella (Martínez Guirao, 2010a, 2010b, 2011), hemos podido apreciar cómo la esfera religiosa aparece difuminada e incluso ausente en el taekwondo que se practica en los gimnasios analizados, y siempre enfrentada a otras esferas, donde el deporte es hegemónico. Si bien es un aspecto que no se ha abordado con profundidad, y que ha quedado pendiente para este y posteriores trabajos.

El artículo no es ni pretende ser en sí mismo una etnografía, ni presentar la estructura propia de la escritura etnográfica, sino realizar un análisis de vínculos entre la religión y el

\footnotetext{
${ }^{7}$ La Kukkiwon es el "gimnasio central" que, entre otros aspectos, en colaboración con la WT, marca las pautas de desarrollo del taekwondo.

8 En el año 2017 la World Taekwondo Federation (WTF) cambió su nombre por World Taekwondo (WT).

${ }^{9}$ La práctica del taekwondo en la provincia de Alicante se remonta al año 1971, cuando se abrió el primer gimnasio en Benidorm. Actualmente acoge la sede de la Federación Española de Taekwondo.
} 
taekwondo a partir, principalmente, de los discursos oficiales extraídos de fuentes documentales, pero teniendo en cuenta los espacios, la cultura material y las prácticas que se realizan en los gimnasios. Es por ello que se hace necesario el carácter descriptivo que facilita la observación, la cual no se ha realizado solamente como practicante, sino en el contexto de un trabajo de campo antropológico, el cual a su vez se hace necesario para la contextualización de los textos analizados, así como para su elección ${ }^{10}$. No obstante, para la redacción de este artículo las fuentes documentales procedentes de instituciones oficiales son el principal material exegético, entendido como el de informantes expertos, es decir como discurso esotérico (Turner, 1980, p. 56).

Con respecto al análisis simbólico, la teoría de Turner establece tres propiedades para los símbolos rituales. La condensación, que implica la multiplicidad de significados que puede evocar cada uno de ellos; la unificación, que refiere a la asociación de todos esos significados; y la polarización de sentido en un polo ideológico y otro sensorial, el primero relacionado con las normas y valores, es decir, con la organización social, y el segundo con la forma externa del símbolo (Turner, 1980, pp. 30-32).

Turner (1980) desarrolló un enfoque metodológico ecléctico para el estudio de los símbolos (Reynoso, 1987, p. 110), que aplica a los procesos rituales. Para Turner (1980, p. 22) los significados de estos símbolos pueden deducirse a partir de la información obtenida de su forma observable, de las exégesis de los practicantes, tanto expertos como inexpertos, y del contexto. Es decir, tiene en cuenta los planos posicional, exegético y operacional. De este modo, la información en el nivel exegético se obtendría desde los discursos de los informantes, con interpretaciones esotéricas cuando las transmiten los especialistas rituales, o exotéricas si provienen de "simples fieles". La operacional vendría de la observación conductual, es decir, sus usos, y la confrontación de los hechos con los discursos, teniendo en cuenta, además de los actos miméticos, la dimensión emotiva y sus manifestaciones, a modo de agresividad, melancolía, gozo, burla, etc. El plano posicional implicaría tener en cuenta la totalidad de símbolos que aparecen y su lugar con respecto a ellos (Turner, 1980, p. 56).

Teniendo en cuenta el objetivo de este artículo, nos centramos, como hemos señalado, en el plano exegético desde fuentes esotéricas, es decir, en la elaboración de todo un entramado simbólico y en discurso construido en torno a él desde las instituciones oficiales. Pero se atenderá a un nivel de análisis distinto al desarrollado por Turner, prestando atención a otros elementos contextuales más amplios, como el proceso en el que se desarrolla esta tradición y las diferencias culturales entre las sociedades que exportan y las que reciben los símbolos. Estos aspectos, sin duda, condicionarán los significados de cada uno de los símbolos en la práctica de los gimnasios estudiados, y serán punto de partida para el análisis de los otros planos que señala Turner, que se realizarán en posteriores trabajos.

\section{Sincretismo y descontextualización de los símbolos religiosos}

El análisis de los elementos religiosos que aparecen en el taekwondo presenta una gran complejidad, debido a la diversidad de textos y religiones de los que se extraen estos conceptos, que se encuentran imbricados de una manera sincrética, procediendo a su vez de sistemas de creencias también sincréticos, los cuales comparten ideas y símbolos, a veces con distintos matices en su interpretación en función de la tradición religiosa. De este modo, en el taekwondo se fusionan, en

\footnotetext{
${ }^{10}$ El trabajo de campo se ha desarrollado principalmente en tres fases: una primera entre los años $2004 \mathrm{y}$ 2008, una segunda en los años 2010 y 2011 y una tercera en 2017 y 2018. Las técnicas utilizadas han sido la observación participante, las entrevistas abiertas y estructuradas, las historias de vida y el grupo de discusión, todas ellas aplicadas en la primera fase, mientras que en la segunda y tercera se llevaron a cabo la observación participante y las entrevistas abiertas. Para ello se seleccionaron cinco gimnasios teniendo en cuenta criterios como el origen del maestro, las actividades que se realizan en el gimnasio o los años de antigüedad. El rol adoptado fue el de investigador, si bien éste es practicante de taekwondo, en otras épocas anteriores a la investigación también ejerció el rol de maestro y de competidor. Es por ello por lo que, además, se pudo proceder a la interpretación de los recuerdos y la experiencia previa como practicante e instructor de taekwondo desde la década de 1980.
} 
diferentes grados, aspectos de los libros clásicos confucianos ${ }^{11}$, además del Lunyu o Analectas de Confucio, el Clásico de la piedad filial (Xiaojing), el Libro de Mencio, libros taoístas como el Daodejing (Libro del Tao) de Laozi, el Zhuangzi, el Liezi, el Huainanzi numerosos sutras budistas (especialmente del budismo zen), el Samguk Yusa coreano, etc.

Además, estos elementos religiosos, son mencionados, simbolizados y se presentan exégesis sobre ellos desde las instituciones oficiales, sin hacer apenas mención a estas fuentes, o sin contextualizarlas dentro de los sistemas religiosos o las culturas de los que proceden, lo cual puede influir en la pérdida de los significados originarios y en su continua reinterpretación, cuando no desaparición de la práctica. El resultado, para algunos autores (Moenig, 2015), es confuso ya en sus orígenes. En efecto, como hemos señalado anteriormente (Martínez Guirao, 2011), hay cierta incoherencia entre el sistema de valores del taekwondo, su simbolismo y su repertorio técnico, que se ha visto acrecentada en las adaptaciones locales o con el propio desarrollo y cambio impulsado desde las federaciones deportivas. Habría que añadir, además, la confusión que resulta cuando se mencionan, en su traducción coreana, términos mayormente difundidos desde el idioma chino, y con diferentes formas de romanización.

Como venimos advirtiendo, uno de los principales aspectos que hace evidente la presencia de las religiones en el taekwondo, se encuentra en la simbología y las explicaciones que se dan en torno a ella. Podemos afirmar que son los elementos simbólicos los que crean los lazos identitarios entre los practicantes de taekwondo, y su relación con las religiones señaladas les hace identificar esta práctica con artes marciales procedentes de Asia Oriental, que presentan peculiaridades que las distanciarían de los deportes de combate occidentales.

Es cierto que los símbolos que aparecen en el taekwondo, de acuerdo con Turner (1980), condensan, unifican y polarizan significados; son a su vez polisémicos, inacabados y dinámicos, pero también construidos, y en este caso, los relacionados con las religiones orientales, desde los discursos oficiales. Sin bien sus formas o significantes no son arbitrarios y en base a ellos se suelen justificar sus significados (Velasco, 2007, p. 19), la multiplicidad de éstos requiere interpretarlos dentro de su contexto sociocultural. Los símbolos presentes en el taekwondo están relacionados con sistemas religiosos muy alejados de los taekwondistas alicantinos. Esto propicia que los trigramas, la forma de la indumentaria, la bandera coreana, los colores de los cinturones, las técnicas o la cruz gamada o esvástica u otros caminos de los pumses ${ }^{12}$, no se perciban como símbolos religiosos, y en todo caso, interpreten desde la cultura propia. Podemos encontrar estos elementos simbólicos en la propia terminología, en la cultura material, en los espacios habilitados para la instrucción, o en las mismas técnicas que se realizan especialmente en los pumses.

\subsection{El do: el camino marcial}

El primer vínculo entre el taekwondo y las religiones lo hallamos en su propia denominación. El sufijo do, que se podría traducir por "camino" o "curso", tiene su correspondiente chino en tao, que suele relacionarse con la religión taoísta. Si bien, hay que tener presente que este concepto no es exclusivo de esta religión y se encuentra en todo el pensamiento chino, y por extensión en las ideas japonesas y coreanas; para Kyong (2000, p. 55) la aplicación del término do al taekwondo es claramente taoísta, pues consiste en seguir el curso de la naturaleza.

En efecto, como afirma Dosset (2000, p. 72), para el taoísmo, el tao o do, se basa en una serie de principios prácticos como el wu wei o no acción, el rechazo al racionalismo, y la búsqueda del equilibrio entre el yin y el yang y los cinco procesos. Su aplicación permitiría vivir en armonía con el do o tao.

Aunque es un término que ya había sido utilizado anteriormente, fue Jigoro Kano quien lo introdujo en el judo y lo popularizó en las artes marciales modernas (Villamón \& Brousse, 1999, p. 110). Como ya han apreciado diferentes autores (Kim, 1999, p. 87; Villamón \& Espartero, 1999, p.

\footnotetext{
${ }^{11}$ Clásico de la poesía (Shijing), Clásico de los documentos (Shujing), Clásico del ritual (Liji), Clásico de la música (Yuejing), Clásico de los cambios (Yijing) y Clásico de las primaveras y otoños (Chunqiu).

12 Los pumses son combinaciones de movimientos técnicos preestablecidos.
} 
55), el sufijo $d o$, además del taekwondo está presente en muchas artes marciales del este asiático, como por el ejemplo el aikido, el bando, el tangsudo, el hapkido, el hwarangdo, el iaido, el jeet kune do, el kendo, el kobudo, el kyudo, etc. Pero su importancia trasciende las artes marciales, y podemos también encontrarlo en otras actividades como el kado (arte floral japonés), el sado (arte de la ceremonia del té) o el shodo (arte de la caligrafía).

Junto a las influencias taoístas, y de toda la cosmología china, en el taekwondo el do condensa estos elementos entremezclados con los propios de las religiones coreanas nacionales. En concreto, Kyong (2000) alude al mito de creación del pueblo coreano y a la figura mitológica de Dangun, al que se considera padre fundador de la nación y que según algunos autores (Dómenech, 2005), es una figura que probablemente esté basada en un rey chamán. Para Kyong (2000), y por tanto para los discursos oficiales, el $d o$ en el taekwondo hace referencia a la "filosofía Tangun" (chamanismo coreano) y a un principio derivado de éste, el de Hongik Ingan, que en líneas generales se basa en la idea de que la relación entre los seres humanos debe regirse por dar y recibir ayuda, ayudando a los demás como se ayudaría uno a sí mismo.

Es significativo cómo el término do está muy presente en el léxico del taekwondo, al aparecer también en la palabra dojang (sala para seguir el camino) y dobok (indumentaria para seguir el camino).

\subsection{El "hábito" marcial}

La indumentaria básica para la práctica del taekwondo está compuesta por un dobok o traje, que ya en su propia denominación incluye el término do (indumentaria para seguir el camino), y un cinturón que simboliza el grado o nivel. A su vez los cinturones se corresponden con los kup (gup), que constituyen un sistema numérico de graduación, que comienza por el 9o kup y acaba en el 1ํ13.

El primer aspecto simbólico del cinturón es el color, que en los manuales oficiales procedentes de Corea se interpreta relacionándolo con el yin y el yang y con los wuxing o cinco procesos ${ }^{14}$, donde no faltan alusiones a las religiones nacionales coreanas y al fundador mítico de la nación. En efecto, como señala Kyong (2000) los cinturones blanco y negro, simbolizan el yin y el yang ${ }^{15}$, es decir, la luz y oscuridad, el día y la noche, el principio y el fin. Posteriormente afirma que el blanco estaría vinculado con el fundador mítico Dangun y con la sacralidad que se atribuía al sol y su luz en "aquella época".

El blanco y el negro se identifican con la filosofía de yin (Um) y del yang: sol y luna, día y noche, principio y fin. El significado del uso de estos colores se puede encontrar en la teoría de Umyang Ohaeng Sol. Um-yang se entiende como el principio último del orden cósmico responsable de la creación de todas las formas de vida que obedecen al principio de acción y reacción. Umyang Ohaeng Sol se basa en la teoría de Umyang que explica la armonía del Yin (Um) y del Yang, teoría que describe el nacimiento y la muerte de cualquier vida a través de los cinco componentes que son el fuego, agua, madera, metal y tierra. El norte, sur, este, oeste y centro se llama Obagsaek. En el ser humano estos elementos se traducen en corazón, riñón, pulmón, hígado y bazo. Los cinco colores en los cinturones de taekwondo se basan en el principio de Oaeng-ogi. Estos colores se utilizan frecuentemente en Corea, en sus diseños, construcciones y ropas (...) Un principiante comienza con el cinturón blanco. Este color tiene un gran significado en la historia coreana. Cuando Tangun, que era hijo de Hwanung, fundó Corea bajo el nombre de Choson, su nombre se basaba en la adoración que se le tenía al sol (simboliza la luz). El color blanco representa el nacimiento o comienzo; además, es el color central en los colores primarios y la base de todos ellos. Representa tanto el comienzo como el final, que no es otra cosa más que el principio de un nuevo comienzo: es el ciclo inevitable de la vida y la muerte. (pp. 45-48)

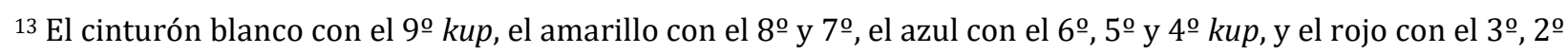
y $1^{\circ}$ (Lee Won Il, 1979, p. 13).

${ }^{14}$ Principios que se recogen en el Clásico de los cambios y que se hayan presentes tanto en el taoísmo como en el confucianismo.

${ }^{15}$ Las denominaciones yin y yang provienen del chino, y en coreano son traducidas y romanizadas como eum y yang, y así se hallan en los textos oficiales del taekwondo. No obstante, para simplificar la lectura y facilitar la compresión se usará en este artículo la terminología china.
} 
Los cinco colores de cinturones que existen en el taekwondo en Corea (blanco, amarillo, rojo, azul y negro), representan los cinco procesos - con los que coinciden en los colores - a modo de ciclo vital que finalizaría con la muerte o cinturón blanco, que es a su vez un nuevo comienzo, aspecto que, aunque está relacionado con el Clásico de los cambios, se vuelve en cierto modo incoherente con la interpretación anterior. A su vez, los nueve kups y nueve danes (sistema que viene tras la consecución del cinturón negro, y que se representa mediante marcas en el cinturón), simbolizan con el número nueve la suma de los cinco procesos más el cielo, la tierra, el yin y el yang.

Esta exégesis oficial resulta incompatible con el desarrollo del taekwondo en los gimnasios alicantinos, debido a que, a su llegada a España, con su inclusión en sus primeros tiempos en la Federación de Judo, adoptó el sistema de cinturones Kawaishi (Martínez Guirao, 2011, p. 230), donde los colores anteriores se sustituyen por el blanco, amarillo, naranja, verde, azul, marrón y negro (Figura 1). Los nueve kups (y por tanto la simbología del número nueve) se ven sustituidos por once, que corresponden a la suma de los cinturones enteros e intermedios (blanco, blanco amarillo, amarillo, amarillo-naranja, etc.).

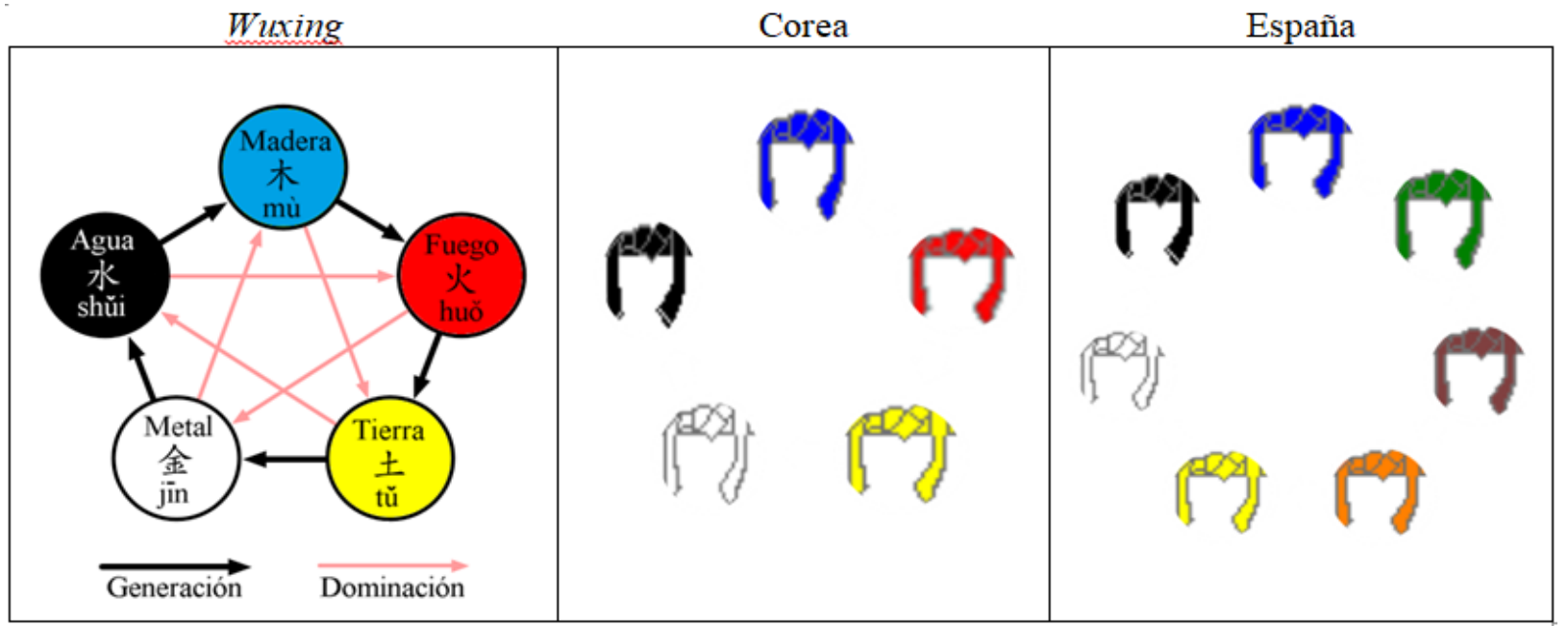

Figura 1. A la izquierda el símbolo del wuxing relacionado con los colores (wuying) según la obra del Zhouyi Cantongqi. Al centro y derecha colores de los cinturones en Corea y en España ${ }^{16}$. Fuente: elaboración propia.

Por otra parte, se alude al dobok vinculándolo con la tríada, cielo-tierra-hombre, a la que se hace mención en el denominado "Gran comentario", atribuido a Confucio, sobre el Clásico de los cambios, además de en otros textos clásicos. De este modo, los pantalones representarían el yin o la tierra, la chaqueta al yang o el cielo y el cinturón al ser humano Kyong (2000). Igualmente se buscan relaciones con las tres figuras geométricas: círculo, cuadrado y triángulo; figuras que se asocian con los principios del cosmos en el libro taoísta Huainanzi (140 a.C.). Así, la línea de la cintura del uniforme es circular y simboliza el cielo, los puños son cuadrados y representan la tierra, y a la altura de la cintura es triangular refiriéndose al ser humano, los tres juntos representan al universo. Este énfasis en el número tres, ya señalado, aparece continuamente buscando clasificaciones que sumen tres elementos en cualquier aspecto del arte marcial.

El origen de las tradiciones coreanas hay que buscarlas en el concepto numérico "Chonbugyong", que contiene los tres principios mencionados: uno para el cielo, dos para la tierra y tres para el hombre. Las tradiciones coreanas han evolucionado al igual que el Dobok, que ha sufrido modificaciones con el tiempo. (...) Los cinturones están divididos en tres categorías, que dependen del nivel de conocimiento que se tenga del arte marcial: el cinturón de color, el de dos colores (rojo y negro) y el negro. Es la trinidad (tres prendas: pantalón, camisola, cinto o los tres colores, de acuerdo al nivel del practicante). En la filosofía oriental, el concepto de trinidad es fundamental en la formación del universo, compuesto por la tierra, el cielo y el hombre, de donde mana toda la vida. El concepto de trinidad es también la parte esencial de

\footnotetext{
16 Nótese aquí que la secuencia de los colores de los cinturones no estaría relacionada directamente con los ciclos de generación y dominación.
} 
Chonbugyong, pero para los coreanos, de estos tres elementos el hombre es el que se considera más importante. (Kyong, 2000, pp. 41-45)

Los nuevos doboks, aprobados por la WT desde el año 2011 para las competiciones de combate, de pumses y de exhibición, que son de nuevos colores (amarillo y azul oscuro, blanco y rojo, blanco y azul, blanco y azul claro, blanco y negro o azul oscuro, azul o rojo) y formas (chaqueta abierta), o la inclusión de pantalones de diferentes colores, trastocan esta construcción simbólica dando pie a nuevas interpretaciones. Si bien podemos inferir que de algún modo nos vuelven a remitir a los colores del wuxing y a los incluidos en el símbolo del sam taeguk ${ }^{17}$ (Figura 2).

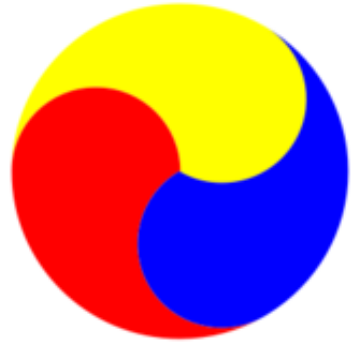

Figura 2. Símbolo del sam taeguk. Fuente: https://es.wikipedia.org/wiki/Taegeuk\#/media/File:Sam Taegeuk (LynneCmix).png

\subsection{El dojang: símbolos en un espacio liminoide}

A diferencia de lo que ocurre con la indumentaria, la simbología presente en la sala de práctica o dojang se hace más explícita, tanto en los aspectos religiosos como en los nacionalistas; pues, como vemos, los referentes religiosos del taekwondo se hallan estrechamente asociados a los nacionales. Dichos símbolos se unen a una actitud ritualizada que confiere a la sala una cierta sacralidad.

La propia denominación de dojang, como sucedía con el término taekwondo, incluye el sufijo do, lo cual resulta indicativo. Aún más si sabemos que el dojang (que procede del japonés dojo) comparte nombre con las salas budistas donde se practica esta religión. Suelen ser espacios relativamente aislados del exterior (al menos en los inicios del taekwondo en Alicante), a los cuales, para acceder, además de ser necesario el permiso correspondiente del maestro o cinturón superior que había que solicitar como inicio del ritual, se realizaba un saludo inclinado (kionne/kyeong-rye) como símbolo de respeto. Este mismo proceso era requerido para salir de ella. Una vez dentro se altera la estructura social y se entra en un tiempo y un espacio que separa al taekwondista de su vida cotidiana, donde se fusionan comportamientos religiosos con militares, donde impera un silencio que le otorgan a la sala un aire de solemnidad, asemejándose a lo que Turner (1977) denominara como espacio liminoide.

En las paredes de algunos gimnasios aparecen, junto a otros motivos orientales, cuadros o textos con los valores que debe seguir el taekwondista y que nos remiten al confucianismo y al budismo. La bandera coreana, que estaba ya en la década de 1970 y todavía se mantiene en las paredes de muchos de estos gimnasios junto a la española, es en sí misma un símbolo religioso además de nacional. Está compuesta por el taeguk o taijitu, imagen de la realidad o principio supremo, en el centro, acompañado por los trigramas que se exponen en el Clásico de los cambios (Figura 3).

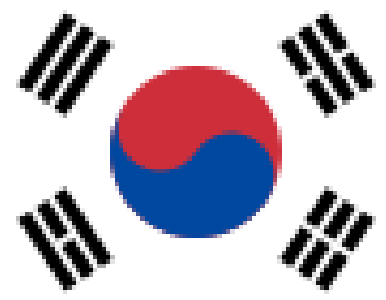

Figura 3. Bandera coreana. Fuente: https://es.wikipedia.org/wiki/Bandera de Corea del Sur\#/media /File:Flag of South Korea.svg

\footnotetext{
${ }^{17}$ El sam taeguk es un símbolo que contiene el color amarillo, junto al rojo y al azul, colores que se relacionan con el ser humano, la tierra y el cielo respectivamente.
} 
Por último, los colores de los tatamis más frecuentes combinan también el azul con el rojo, o con el amarillo, tres de los cinco colores con los que se asocian los wuxing o lo tres del sam taeguk (variante tricolor del taeguk).

\subsection{Los pumses}

Además de los símbolos de la cultura material, el repertorio técnico condensa igualmente multitud de elementos simbólicos. Los pumses, combinaciones de movimientos técnicos preestablecidos, presentan simbología relacionada con el Clásico de los cambios y las religiones que se apoyan en estas ideas (confucianismo y taoísmo), con el budismo y con las religiones nacionales. Como se desprende de las interpretaciones de los manuales oficiales de Kim Boo Hyang (1989) y Kyong Myong Lee (2000), los ocho pumses taeguk (taegeuk) representan los trigramas del libro de los cambios (por este orden: cielo, lago, fuego, trueno, viento, agua, montaña y tierra), secuenciados siguiendo los principios del yin y el yang, lo que a su vez se pretende que quede manifestado en sus técnicas.

Por su parte, los pumses superiores ${ }^{18}$ se centran en representar aspectos históricos, mitológicos, geográficos y también religiosos relacionados con la nación coreana. Así, uno de ellos adquiere el nombre de una dinastía histórica, otro lleva el nombre de una montaña sagrada donde los mitos dicen que se fundó la nación coreana, otro simboliza la tierra, otro el cielo, etc. El último pumse, el ilyo, representa el despertar budista o nirvana y tal y como demuestra el dibujo de la cruz gamada que marca en el suelo su recorrido (figura 4).

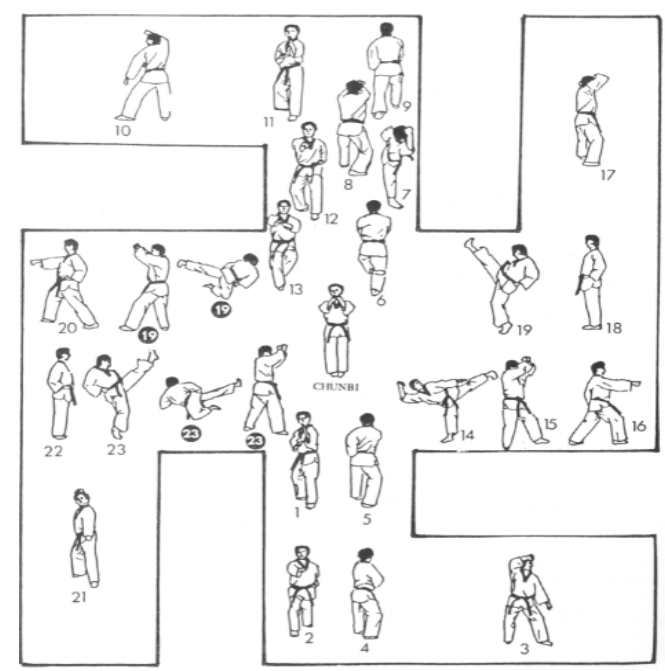

Figura 4. Dibujo del recorrido en el suelo del pumse ilyo. Fuente: Kim (1989, p. 206).

\section{Influencias prácticas religiosas: el ki y sus vías de desarrollo}

Como hemos visto en el apartado anterior, los aspectos religiosos trascienden los elementos simbólicos para tratar de repercutir en la propia práctica en el repertorio técnico. Un ejemplo claro de ello, propio de las artes marciales procedentes de Asia Oriental, es el concepto ki, también conocido en su traducción del chino como chi.

Según Jones (2003, p. xii), el ki es un componente animatista que se encuentra en la mayoría de las artes marciales y que consiste en la creencia en una energía o fuerza interna que puede dotar a la persona de cualidades casi sobrehumanas. El ki sería un principio fundamental en todas las religiones mencionadas, no sólo como energía interna, sino que estaría presente en todo el universo. Es pues el principal elemento que ha permitido que se construyera una imagen esotérica y mística en torno a ellas tan claramente representada por el cine de artes marciales. No obstante, en el caso del taekwondo el ki no se muestra tan explícito como en otras artes marciales, pues en algunas de ellas, al igual que sucede con el sufijo $d o$, se halla en la misma denominación (tal es el caso del aikido, el hapkido, etc.).

\footnotetext{
18 Los pumses superiores se realizan a partir del cinturón negro y son diez: koryo, kumgang (Keumgang), taebaek (taebek), pyongwon, sipjin (shipjin), jitae, cheongkwon (chonkwon), hansu e ilyo.
} 
Aun así, los manuales oficiales (Kyong, 2000) o incluso la propia Federación Coreana (Korea Taekwondo Association KTA) nos hablan de que esta energía se puede controlar, denominando a la manera de hacerlo como tanjon hohup o shimgi, que se basa en ejercicios de respiración y meditación. Según estos autores, el lugar del cuerpo donde se concentra esta energía se localizaría entre cinco y diez centímetros por debajo del ombligo, en un punto llamado tanjon, que sería equivalente al hara japonés ${ }^{19}$, la función de estos ejercicios es canalizarlo a través de él.

En nuestra vida cotidiana, el mantenimiento del cuerpo funciona a costa de energía. En el entrenamiento de taekwondo, esa fuente de energía es el Ki. El Ki se puede desarrollar mediante el Tanjon Hohup, que es una manera de vivenciar la energía que se obtiene y poder controlarla y canalizarla a través de la actividad mental. Comúnmente a este concepto también se le conoce como "Shimgi" (...) El Shim está en constante reposo (Chong) y el Ki está en continua acción (Tong). En el taekwondo estos dos conceptos deben formar una sola unidad. Cuando el Ki se domina a la perfección, es sorprendente la cantidad de fuerza que se puede disponer. (Kyong, 2000, p. 53)

Se confiere, a su vez, esta función al cinturón, afirmando que conecta la parte inferior y superior del uniforme cubriendo el centro del cuerpo, y que de este modo puede ayudar a llevar la energía al músculo que conecta un punto de acupuntura que se ubica en la cintura, denominado meongmunhyul, y el centro corporal de la zona inferior del abdomen (Kukkiwon, 2018a).

La meditación se halla presente tanto en el taoísmo como en el budismo, en especial en el budismo zen, en el cual es además la práctica fundamental para alcanzar el satori o "despertar". Con variaciones según la escuela y tradición religiosa, la meditación puede realizarse en el suelo de manera estática o en movimiento. En este último sentido, siguiendo a autores como Langsner (2003, p. 1), se podría considerar a los pumses como una forma de meditación en movimiento. Si prestamos atención a las técnicas que incluyen, vemos que algunas de ellas consisten en movimientos de preparación o respiración, idea que se refuerza si los interpretamos unidos a su exégesis oficial. Esto resulta mucho más evidente en artes marciales como el tai chi, que se basan en las formas como medios para canalizar el $k i$.

Otra forma de canalizar esa energía, es el kiap o grito, que consiste en la proyección del ki mediante el sonido. La mayor parte de las técnicas del taekwondo van unidas al kiap si bien, dentro del contexto del combate se prescinde habitualmente de éste.

\section{El código de valores y el ritualismo}

En lo que respecta a los valores ${ }^{20}$, los discursos oficiales (Kukkiwon, 2018b) hacen referencia al código de conducta de los guerreros Hwarang del reino de Silla (57 a.C.-668 d.C.), y los relacionan directamente con el budismo, el confucianismo y las religiones coreanas.

En efecto, el código moral Hwarang o Hwarangdo se estructura en tres virtudes, tres conductas virtuosas y cinco principios, que constituyen una mezcla de valores que comparten con las religiones señaladas. Es decir, valores confucianos como la lealtad al país, al rey y la nación, el respeto a los padres y la piedad filial o la fidelidad a los amigos; budistas como la humildad, la frugalidad, la moderación, la no violencia; o propios de las religiones coreanas como la fe y la confianza en los seres humanos. De estas últimas se desprenden también los principios que señala la Kukkiwon (2018b) del hongik-ingan y jaese ihwa, el primero prescribe la obligación de la búsqueda del bienestar de todos los seres humanos, el segundo actuar siguiendo el $d o$, que según

\footnotetext{
${ }^{19}$ Conocido popularmente por estar incluido en el término harakiri.

20 El código de valores del taekwondo impulsado desde las instituciones oficiales, no aparece claramente expuesto o sintetizado y resulta de un sincretismo entre diferentes religiones y el deporte. Además de los valores propios del deporte (la competición, la autosuperación, la igualdad de oportunidades, el juego limpio, etc.), en el manual de Kyong Myong Lee (2000) aparecen valores como el altruismo, la intrepidez, el autodominio o el ritualismo. Kim Boo Hyan (1989) por su parte alude al respeto a la jerarquía y a los símbolos. Tanto en ambos textos como en las páginas de la Korea Taekwondo Association (KTA), la Kukkiwon y la World Taekwondo (WT), se hace alusión al código Hwarang y a los principios del hongik-ingan y jaese ihwa, que se exponen a continuación.
} 
señalaba la World Taekwondo (2007) implicaría seguir el mandato del Cielo, idea amplia que aparece habitualmente en el confucianismo.

El respeto a una estructura jerárquica muy rígida, basada en el cinturón y en la antigüedad, junto a otros elementos como la edad, se ha visto impulsada también desde las instituciones oficiales. El manual de Kim Boo Hyang (1989, pp. 21-25) prescribe cómo deben regirse tanto las relaciones entre los taekwondistas, los comportamientos que han de extrapolarse a situaciones que acontezcan fuera del gimnasio, o cómo se debe actuar en situaciones frecuentes en las propias vidas cotidianas. Estos aspectos se derivan del confucianismo, y tratan de aplicarse de manera descontextualizada en sociedades con sistemas de valores diferentes, e incluso incompatibles, pues sitúan a la sociedad por encima del individuo, algo propio de lo que Dumont (1970) definiría como sociedades holistas.

Otro elemento muy relacionado con la tradición confuciana es el ritualismo que se ha tratado de conferir tanto a la práctica del taekwondo como a las relaciones interpersonales. En la práctica del taekwondo hay prescritos saludos rituales al maestro, entre los alumnos, a la bandera, al dojang, secuencias antes de iniciar la clase o de finalizarla, al entregarse un objeto, etc. Este importancia que se da al ritual tendría también sus orígenes en la oposición confuciana al legismo ${ }^{21}$, y el énfasis del ritual sobre la ley para preservar el orden en la sociedad.

\section{Consideraciones finales}

Desde la perspectiva oficial la esfera religiosa resulta evidente en el taekwondo, hasta el grado de que éste podría entenderse como una actividad encaminada incluso a alcanzar el propio nirvana budista. Esta oficialidad ha dotado al taekwondo de un complejo sistema de símbolos y mensajes filosóficos relacionados con religiones como el budismo, el taoísmo, el confucianismo y otros sistemas de creencias coreanos, pero que, como hemos visto, presenta incoherencias y contradicciones tanto a nivel interno como en sus procesos de adaptación cultural. Así, para comprender la importancia y el desarrollo de estos aspectos, hay que tener presente el proceso globalizador y las diferentes implementaciones y adaptaciones culturales del taekwondo. Igualmente debemos prestar atención a las diferentes configuraciones que puede adquirir el taekwondo, en las que su vertiente deportiva es hegemónica. La visión del taekwondo como deporte ha tenido incidencia en la propia categoría de religión.

A diferencia de lo que ha ocurrido con las federaciones coreanas, la Federación Española de Taekwondo se ha centrado en la esfera deportiva y en los aspectos del taekwondo compatibles con el deporte, omitiendo cualquier alusión a la religión, tanto en su página web como en los manuales avalados por ella, y lo mismo sucede con cada una de las doce federaciones regionales que disponen de sitio $w_{e b}{ }^{22}$. Y en los cursos para monitores y entrenadores de taekwondo se elude incluir temáticas relacionadas con la filosofía y la religión.

Esta tendencia parece haber afectado incluso a la propia WT que, con la remodelación de su página web en el año 2016, prescindió de los comentarios sobre los elementos religiosos. Pero en cambio impulsó iniciativas como el World Taekwondo Peace Corps (TPC), que se creó en el año 2008 para continuar e intensificar la difusión internacional del taekwondo con el lema "La paz mundial a través del gran espíritu del taekwondo", con la intención de "difundir una de las más grandes herencias espirituales de Corea, 'el Espíritu del Taekwondo', trabajando en todo el mundo con los valores de ese espíritu para difundir la paz" (WT, 2018). Algo similar ha ocurrido recientemente en la página web de la KTA, enfocada a Corea. Tan sólo la Kukkiwon parece seguir manteniendo estos aspectos. De este modo, vemos cómo el constructo religioso se desvanece cada vez más incluso en las propias instituciones que lo han fomentado.

\footnotetext{
${ }^{21}$ El legismo es una corriente de pensamiento que se basa en la ley, y quedó recogido en China en obras como el Guanzi, el Libro del Señor Shang, o el Hanfeizi (Prevosti, 2005, pp. 63-64). En la historia de China ha habido disputas entre las ideas legistas y confucianistas, con hegemonía de estas últimas.

22 Federación Aragonesa, Federación de Euskadi, Federación Balear, Federación Madrileña, Federación Valenciana, Federación Castellano-Leonesa, Federación Navarra, Federación Murciana, Federación Catalana, Federación Canaria, Federación Andaluza y Federación Gallega.
} 
Para posteriores trabajos queda profundizar en la esfera religiosa en la práctica de los gimnasios alicantinos, analizar desde un punto de vista diacrónico el modo como los elementos religiosos aparecen o han aparecido en los valores, la jerarquía y los rituales de interacción social, así como las diferencias entre las exégesis de los elementos simbólicos que se pueden producir entre propios taekwondistas y los discursos oficiales.

\section{Referencias}

Bowman, P. (2010). Theorizing Bruce Lee. Film-Fantasy-Fighting-Philosophy. Amsterdam \& New York: Rodopi.

Bowman, P. (2013). Beyond Bruce Lee: Chasing the Dragon through Film, Philosophy, and Popular Culture. London \& New York: Wallflower Press.

Bowman, P. (2015). Everything you know about taekwondo. Conference Martial Arts and Traditional Sportsin Asia: History, Politics and Culture, Muju, South Korea, 21-22 November. Recuperado https://www.academia.edu/12257421/Everything you know about Taekwondo

Bowman, P., \& Judkins, B.N. (2016). Editorial. Martial Arts Studies, (2), 1-5. doi: $10.18573 / \mathrm{j} .2016 .10061$

Brown, D.H., Jennings, G., \& Molle, A. (2009) Belief in the martial arts: Exploring relationships between Asian martial arts and religion. Stadion. International Journal of the History of Sport, $35,47-66$.

Burdick, D. (1997). People and Events of T'aekwondo's Formative Years. Journal of Asian Martial Arts. 6(1), 30-49.

Capener, S. (1995). Problems in the Identity and Philosophy of Taekwondo and Their Historical Causes. Korea Journal, 35(4). Recuperado de https://www.americanmoodokwan.com/Capener Essay(W-95).pdf

Capener, S. D. (2016). The Making of a Modern Myth: Inventing a Tradition for Taekwondo. Korea Journal, 56(1), 61-92.

Consejo Superior de Deportes. (2017). MEMORIA 2017/ Licencias y Clubes federados. Recuperado de http://www.csd.gob.es/csd/estaticos/asoc-fed/licencias y clubes 2017.pdf

Corbetta, P. (2007). Metodología y técnicas de investigación social. Madrid: McGraw Hill.

Cynarski W. J. (2001). Commercialization Process of the Far East Martial Arts. Studies in Physical Culture and Tourism, (8), 191-201.

Cynarski W. J. (2004). Teoria I praktyka dalekowschodnich sztuk walki w perspektywie europejskiej [Theory and practice of Far-Eastern martial arts in a European perspective. Wydawn: Uniwersytetu Rzeszowskiego.

Cynarski, W. J., Sieber, L., \& Litwiniuk, A. (2006). Azjatyckie sztuki walki w oord.a europejskiej I amerykańskiej [Asian Martial Arts in the European and American reception]. Ido Movement for Culture. Journal of Martial Arts Anthropology, (6), 252-260.

Dohrenwend R. E. (2000). Informal History of Chung Do Kwan Tae Kwon Do. Recuperado de http://husky.students.mtu.edu/cdk.shtml

Doménech, A. J. (2005). Religiones autóctonas de Asia oriental: Japón y Corea. Shintoísmo y Chamanismo. En A. Prevosti (Coord.), Pensamiento y religión en Asia oriental (pp. 233-281). Barcelona: UOC.

Donohue, J. J. (1994). Wave People: The Martial Arts and the American Imagination. Journal of Asian Martial Arts, 3(1), 10-25.

Dosset, W. (2000). Tradiciones de Asia Oriental. En N. Smart (Ed.) Atlas de las Religiones (pp. 6885). Colonia: Koneman Verlagsgesellschaft mbH.

Draeger, D. F., \& Smith, R. W. (1969.) Asian Fighting Arts. Tokyo \& Palo Alto: Kosansha International.

Dumont, L. (1970). Homo hierarchicus. Ensayo sobre un sistema de castas. Madrid: Aguilar.

Erlandson, D. A., Harris, E. L., Skipper, B. L., \& Allen, S. D. (1993). Doing Naturalistic Inquiry. London: Sage.

Ferreira, F. D. C., Sonoda-Nunes, R. J., De Almeida, B. S., De Oliveira, S. R., \& Marchi Junior, W. (2012). A Sociological View Over the Present Scenery of Kung Fu in the Occident: A Comprehensive Panorama Over the Chinese Martial Arts in Brazil. Ido Movement for Culture. Journal of Martial Arts Anthropology, 12(1), 2-6. 
Frank, A.D. (2000). Experiencing Qi. Text, Practice, Perfomance, 2, 13-31.

Frank, A.D. (2006). Taijiquan and the Search for the Little Old Chinese Man: Understanding Identity through Martial Arts. Hampshire: Palgrave Macmillan.

Gutiérrez-García, C. (2004). Introducción y desarrollo del judo en España: (de principios del siglo XX a 1965): el proceso de implantación de un método educativo y de combate importado de Japón. León: Universidad de León.

Gutiérrez-García, C. (2006). Soldados, samuráis y sportmen: el japonismo deportivo llega a Europa. En Sport and violence (pp. 115-123): Sevilla: Universidad Pablo de Olavide.

Gutiérrez-García, C. (2007). Estudio de las primeras obras sobre artes marciales escritas en español. Revista de Artes Marciales Asiáticas, 2(1): 8-27. doi: 10.18002/rama.v2i1.281

Gutiérrez-García, C., Pérez-Gutiérrez, M., Acevedo, W., y Cheung, M. (2010). Los luchadores japoneses tienen más destreza y arte que los luchadores chinos, o cuando el contexto importa más que el texto. Revista de Artes Marciales Asiáticas, 5(1): 41-52. doi: 10.18002/rama.v5i1.123

Hobsbawn, E. (2002). Introducción: la invención de la tradición. En E. Hobsbawn y T. Ranger (Eds.), La invención de la tradición (pp. 7-23). Barcelona: Crítica.

Jennings, G. (2016). Ancient Wisdom, Modern Warriors. The (Re)Invention of a Mesoamerican Warrior Tradition in Xilam. Martial Arts Studies, (2), 59-70. doi: 10.18573/i.2016.10064

Jimenez-Landazuri, A., Gomez-Alonso, M. T., Izquierdo, E., \& Gutierrez-Garcia, C. (2016). Research into the history of martial arts and combat sports in Spain: The Noticiarios y documentales (No-Do - News newsreels (1943-1981). IDO Movement for Culture. Journal of Martial Arts Anthropology, 16(4): 11-20. doi: 10.14589/ido.16.4.3

Jones, D. E. (2002). Introduction: Toward a Definition of the Martial Arts. En D.E. Jones (Ed.), Combat, Ritual and Performance. Anthropology of Martial Arts (pp. XI-XIV). London: Praeger Publishers.

Jones, D.E. (Ed.). (2002). Combat, Ritual and Performance. Anthropology of Martial Arts. London: Praeger Publishiers.

Kim, B. K. (1989). Taekwondo: Fundamentos y pumses. Barcelona: Dae Do Internacional.

Kim. M. H. (1999). L'Origine et le développement des arts martiaux. Pour une anthropologie des techniques du corps. Paris: L'Harmattan.

Kukkiwon (2012). Taekwondo Textbook. Seúl: Korean Book Service.

Kukkiwon (2018a). Taekwondo Dobok \& Tti. Recuperado de http://www.kukkiwon.or.kr/front/pageView.action?cmd=/eng/information/history unifor $\underline{m}$

Kukkiwon (2018b). Taekwondo philosophy. Recuperado de http://www.kukkiwon.or.kr/front/pageView.action?cmd=/eng/information/taekwondo mi $\underline{\text { nd }}$

Kyong, M. L. (2000). Taekwondo dinámico. Barcelona: Hispano-Europea.

Langsner, M. (2003). A Performance Theory Analysis of the Practice of Kata in Karate-Do: Self Resolving Contradictions of Ritual, Spontaneity, Violence, and Morality. Brandeis Graduate Journal, 1(1). Recuperado de https://ejmas.com/pt/2006pt/ptart langsner 1206.html

Lee Won-Il (1979). Taekwondo. Técnicas superiores. Barcelona: Editorial Hispano-Europea.

Lefebvre, A. (2016). The Pacific Philosophy of Aikido: An Interactional Approach. Martial Arts Studies, (2), 91-109. doi: 10.18573/j.2016.10066

Martínez Guirao, J. E. (2010a). El cuerpo en las artes marciales. Confluencias y divergencias entre Oriente y Occidente. En J. E. Martínez Guirao y A. Téllez (Eds.), Cuerpo y cultura (p. 109-132). Barcelona: Icaria.

Martínez Guirao, J. E. (2010b). La construcción del arte en las artes marciales. Una aproximación antropológica al taekwondo. Gazeta de Antropología, 26(2). Recuperado de http://digibug.ugr.es/handle/10481/6767

Martínez Guirao, J. E. (2011). Una etnografía de las artes marciales. Procesos de cambio y adaptación cultural en el taekwondo. Alicante: Ecu.

Mason, P. H. (2016). Fight-Dancing and the Festival Tabuik in Pariaman, Indonesia and lemanjá in Salvador da Bahia, Brazil. Martial Arts Studies, (2), 71-90. doi: 10.18573/j.2016.10065

McBride, R. D. (2010). Silla Buddhism and the Hwarang. Korean Studies, 34, 54-89. 
Moenig, U, Kim, M. (2016). The Invention of Taekwondo Tradition, 1945-1972: When Mythology Becomes 'History'. Acta Koreana, 19(2): 131-164.

Moenig, U. (2015). Taekwondo: From a Martial Art to a Martial Sport. London: Routledge.

Pérez-Gutiérrez, M., Brown, D. H. K., Álvarez-Del-Palacio, E., y Gutiérrez-Garciá, C. (2015). The (Re)emergence of a religio-spiritual self-cultivation focus in Asian martial arts monographs published in Spain (1906-2009). International Journal of the History of Sport, 32(2): 200-217. doi: $10.1080 / 09523367.2014 .943735$

Prevosti, A. (2005). Fundamentos religiosos de Asia Oriental. Los orígenes de la cultura china. En A. Prevosti, (Coord.), Pensamiento y religión en Asia oriental (pp. 25-73). Barcelona: Editorial UOC.

Reynoso, C. (1987) Paradigmas y estrategias en antropología simbólica. Buenos Aires: Ediciones Búsqueda.

RTVE (2018). Filmoteca Española. NODO. Recuperado de http://www.rtve.es/filmoteca/no-do/

Ruiz Olabuénaga, J. I., \& Ispizua, M. A. (1989). La descodificación de la vida cotidiana. Métodos de investigación cualitativa. Bilbao: Universidad de Deusto.

Turner V. W. (1980). La selva de los símbolos. Madrid: Siglo XXI.

Turner, V. W. (1977). Variations on a Theme of Liminality. En S. F. Moore \& B. G. Myerhoff (Eds.), Secular Ritual (pp. 36-52). Assen/Amsterdam: Van Gorcum.

Velasco, H. (2007). Cuerpo y espacio. Símbolos y metáforas, representación y expresividad de las culturas. Madrid: Editorial universitaria Ramón Areces.

Villamón, M., \& Brousse, M. (1999). Evolución del judo. En M. Villamón, (Dir.), Introducción al judo (pp. 103-145). Barcelona: Hispano Europea.

Villamón, M., \& Espartero, J. (1999). La lucha en Oriente: el significado del "do". En M. Villamón (Dir.), Introducción al judo (pp. 55-103). Barcelona: Hispano Europea.

World Taekwondo (WT) (2007). Philosophy of Taekwondo. Recuperado de http://www.wtf.org/about_tkd/philo_tkd.htm [página web no accesible actualmente]

World Taekwondo (WT) (2018). Taekwondo Peace Corps. Recuperado de http://www.worldtaekwondo.org/development/tpc

\section{Author's biographical data}

Javier Eloy Marínez Guirao (Spain). Profesor en el área de Antropología Social, en el Departamento de Ciencia Política, Antropología Social y Hacienda Pública de la Universidad de Murcia (España). Doctor en Antropología Social y Cultural por la UNED y doctor en Sociología por la Universidad de Alicante. Ha desarrollado su investigación en el campo de la Antropología de las artes marciales y la Antropología del cuerpo, de la que se derivan diversas publicaciones. Practicante de taekwondo desde hace más de 30 años, además de otras artes marciales y deportes de combate como el hapkido y el kickboxing. 